This item was submitted to Loughborough's Research Repository by the author.

Items in Figshare are protected by copyright, with all rights reserved, unless otherwise indicated.

\title{
Scan posture definition and hip girth measurement: the impact on clothing design and body scanning
}

PLEASE CITE THE PUBLISHED VERSION

https://doi.org/10.1080/00140139.2016.1251621

\section{PUBLISHER}

(C) Taylor \& Francis

\section{VERSION}

AM (Accepted Manuscript)

\section{PUBLISHER STATEMENT}

This work is made available according to the conditions of the Creative Commons Attribution-NonCommercialNoDerivatives 4.0 International (CC BY-NC-ND 4.0) licence. Full details of this licence are available at: https://creativecommons.org/licenses/by-nc-nd/4.0/

\section{LICENCE}

CC BY-NC-ND 4.0

\section{REPOSITORY RECORD}

Gill, Simeon, and Christopher J. Parker. 2019. "Scan Posture Definition and Hip Girth Measurement: The Impact on Clothing Design and Body Scanning”. figshare. https://hdl.handle.net/2134/33471. 


\title{
Scan Posture Definition and Hip Girth Measurement: The impact on clothing design and body scanning
}

\begin{abstract}
Ergonomic measurement is central to product design and development; especially for body worn products and clothing. However, there is a large variation in measurement definitions, complicated by new body scanning technology that captures measurements in a posture different to traditional manual methods. Investigations of hip measurement definitions in current clothing measurement practices supports analysis of the effect of scan posture and hip measurement definition on the circumferences of the hip. Here, the hip girth is a key clothing measurement that is not defined in current body scanning measurement standards. Sixty-four participants were scanned in the standard scan posture of a [TC $]^{2}$ body scanner, and also in a natural posture similar to that of traditional manual measurement collection. Results indicate that scan posture affects hip girth circumferences, and that some current clothing measurement practices may not define the largest lower body circumference. Recommendations are made concerning how the hip is defined in measurement practice and within body scanning for clothing product development.
\end{abstract}

Practitioner Summary: The hip girth is an important measurement in garment design, yet its measurement protocol is not currently defined. We demonstrate that body posture during body scanning affects hip circumferences, and that current clothing measurement practices may not define the largest lower body circumference. This paper also provides future measurement practice recommendations.

Keywords: Body Scanning; Measurement; Scan Posture; Clothing; Hip Girth, Clothing Design

Word Count: 5,963

\section{Introduction}

Human measurement is the basis for creating well-fitting and functioning clothing and is central to the communication of garment size. Body measurements required for clothing are detailed in international standards (ISO 1989; BSI 2002) and applied in pattern construction techniques (Beazley and Bond 2003; Aldrich 2008) and often taken in postures different to those recommend for body scanning. With the introduction of body scanning there are new opportunities to gather detailed measurements from the human body (Bye, LaBat, and Delong 2006; Bonnechère et al. 2014) and new standards for measurements captured through body scanning (BSI 2010; ISO 2010). However, there is currently no definition in these standards for the hip circumference or recognition of scan postures potential impact on measurement and different ergonomic disciplines propose different methods for defining the 
hip measurement manually (Clauser et al. 1988; Pheasant 1990; Beazley 1997; A. Stewart et al. 2011; Roebuck 1995).

This research investigates hip circumference definitions in clothing measurement practices and uses definitions derived from analysis to create parameters for measurement extraction through body scanning. A sample population is analysed to establish the impact of scan measurement posture, and measurement definitions on circumferences of the hip. Despite there being many national surveys utilising body scanning, little is known about accuracy and comparability of body scanning measurements. This paper addresses body scanner measurement accuracy as well as considering measurement definition and posture in relation to the hip and raises important questions regarding the effect of scan posture on measurements.

The hip represents one of the key sizing dimensions in pattern construction and sets the largest lower body circumference within the control section (starting at the waist) for garments that fit the lower body (Gill 2015). The hip is also used in sizing communication, and within sizing systems is graded $\pm 4-5 \mathrm{~cm}$ between sizes (Gill and Brownbridge 2013). Graded size difference is similar to the ease allowance in basic fitted and semi fitted pattern blocks (Beazley and Bond 2003; Gill 2015) and instances where measurement methods result in recording of a hip measurement smaller than the largest hip dimension could compromise the effectiveness of ease in creating the correct fit. Product development practices use a single hip measurement and it would be expected to be the largest lower body dimension around which a garment covering the lower body would fit. The hip level for clothing has generally been determined in relation to the anatomical markers of the trochanteric projections (Kemsley 1957; ISO 1989), though the ASTM suggest the projection of the buttocks as a landmark (ASTM 2009). However, body scanning as a non-contact measurement technology does not always allow for the use of anatomical landmarks (Kouchi and Mochimaru 2011), and requires the adoption of a posture different to that used in manual surveys; a factor that has been demonstrated to impact on measurement dimensions (Chi and Kennon 2006; Mckinnon and Istook 2002; Y. J. Wang et al. 2011; Choi and Ashdown 2011). It is important to determine the largest circumference, so the correct data can be used to drive product development, especially in the realisation of mass customisation and garment personalisation.

Body scanning technology provides the opportunity to collect data using definitions which would be difficult to replicate manually, and to collect many more measurements, more quickly, and in most cases with higher reliability than manual methods (Loker, Ashdown, and Schoenfelder 2005; Gill 2015). However it is difficult to consistently define a hip location from the body surface features as the hip region may be defined as between the crotch and waist, but shape in this region may vary due to variation in fat deposition on the female figure, corresponding in many cases to size and age (J. Wang et al. 2003; A. D. Stewart et al. 2010; Alexander, Pisut, and Ivanescu 2012). 


\subsection{The Research Gap}

It is very difficult to achieve the standardisation of body measurement methods throughout the range of disciplines applying ergonomic measurements. This is compounded by technology such as body scanning, where, even when the introduction of standards provides some clarity (BSI 2010), methods are still grounded in historic manual practices (Tyler, Mitchell, and Gill 2012; Li et al. 2008) which do not translate well into the non-contact environment of body scanning (Kouchi and Mochimaru 2011). The latest standard for body scanning measurement (ISO 2010) does not provide a definition for the hip measurement - a key sizing dimensions in clothing and body worn products - and suggests a need for more understanding of this area. Body scanning provides an opportunity to understand measurement of the hip and the impact of varied definition and posture on hip girths determined by different criteria.

Whilst standards exist to help define measurements of the body for clothing (ASTM 2009; ISO 1989), body scanning standards aligned more to ergonomic requirements (ISO 2010), which as noted do not define the hip. Further there are limited details regarding the bust and waist. This lack of clear consistent definition for the hip and other dimensions is mirrored in the variation in manual definitions for body measurements (ISO 1989; Roebuck 1995; A. Stewart et al. 2011; Kunick 1984; Pheasant 1990; Beazley 1997). Whilst creating clothing is only one of the applications of ergonomic measurement of the human body, the variation could be reduced by consideration of measurement purpose, to measurement use in product development (Gill 2009).

\subsection{Study Aims}

The aims of this study are:

1. To establish the effect of posture on the hips' circumference measurements (girths)

2. To establish the waist-to-hip lengths between different hip definitions, and the effect of posture on their position.

3. To establish the most appropriate hip definition to be used in body scanning for product development

4. To determine the effects of posture, related to foot positioning, on hip measurements and any needs for further standardisation of practice.

\subsection{Background Literature}

\subsubsection{Measurement and the Clothing Pattern}

Many of the pattern construction guides used to create basic clothing shapes provide details on body measurement, though these differ in detail and depth (Gill 2009). These measurement guides, however, relate directly to the pattern construction methods and the potential accuracy of the pattern block prior to fit testing and manipulation. Analysis of hip measurement definitions, show some sources provide clear details of placement (Kunick 1984; Beazley and Bond 2003), whilst others are subject to the interpretation of the reader (Haggar 2004; Aldrich 2008). This variation can also be seen in other sources (ISO 1989; 
Roebuck 1995; A. Stewart et al. 2011) highlighting that even with consistent applications (clothing), measurement definitions may vary in detail and depth. Research has established that variation in methods used for defining the waist circumference can lead to considerable waist measurement variation (Gill 2011; Daniell, Olds, and Tomkinson 2010; J. Wang et al. 2003; A. D. Stewart et al. 2010; Gill et al. 2014). Whilst circumference measurements may vary by different definitions, there is little research focused on the hip girth and the impact of hip measurement placement on values returned. Some pattern construction guides suggest a set waist to hip distance, usually around $20 \mathrm{~cm}$, to be applied during the draft process (Beazley and Bond 2003; Aldrich 2008; Kunick 1984) whilst others suggest measurement placements related to body landmarks (Knowles 2005; MacDonald 2010). Studies undertaken into the application and context of measurements within pattern construction by McKinney et al. (2012) and Gill and Chadwick (2009) show how an appreciation of measurement definition and application can help in understanding and controlling variations in practice.

\subsubsection{Body scanning and measurement}

Simmons (2001) reviewed body scanning measurement definitions and found variation in methods to define measurements between different body scanner manufactures. Both the waist and hip were defined in relation to different anatomical markers, related to surface geometry due to the non-contact nature of the body scanners reviewed. Due to the nature of non-contact methods, it is not unusual for those collecting scans to default to manual methods and pre-landmark their participants prior to scanning (Ashdown and $\mathrm{Na} 2008$; Choi and Ashdown 2011). Landmarking accuracy between body scanning and manual methods undertaken by Kouchi and Mochimaru (2011) indicated that traditional landmarks reliant on palpation are not suitable for automated scanning measurement extraction. Whilst initial work by Paquette et al. (2000) suggests automated systems can often provide comparable measurements to those collected manually.

Wang et al. (2003) and Bigaard et al. (2005) discuss waist circumference definition and its variation. Wang et al. (2003) compared manual measurement between four waist locations, though not the same four as investigated in Bigaard et al. (2005). Both sets of authors also agree with ISO 7250 (ISO 2010), that the waist should occur between the top of the iliac crest and lowest palpable rib (see Figure 1). This is in accordance with medical definitions, and suggests landmarks that are easier and more consistent to locate within the population.

Wang et al. (2003) suggests that a single skeletal landmark may be more appropriate, it is also indicated by Bigaard et al. (2005) that natural tapering of the body causes larger circumferences to be found in the lower section of this region, closer to the iliac crest. Referencing findings to skeletal anatomy indicates the structure of the rib cage which tapers in and iliac which widens (Kapandji 1987) would cause these larger lower circumferences. Heuberger et al. (2008) used skin markings to match manual and scan measurements when looking at body scanning to define waist-to-hip ratios. Here methods of determining measurements are grounded in manual practice, and the hip is defined as the widest point passing over the buttocks. 
When defining the hip Alexander et al. (2012) analysed the shape of the lower torso area found in Size USA scan data and note that as body size increases so the greater fullness of the lower body often occurs higher on the portion of the body where the hip would be defined. This paper shows the potential applications of body scanning, though definitions of measurements are not reported, making it difficult to determine hip placement. Han et al. (2010) developed algorithms to place measurements, highlighting the disjointed nature of scan and manual methods and the difficulties of trying to define scan measurements using manual definitions as the benchmark. Body Scanning measurements have also been used by Lee et al. (2007), comparing body shapes between a US and Taiwanese population and documenting the FFIT shape system which uses algorithms related to the proportional relationships between bust, waist, top hip and hip to classify participants by one of seven shapes.

\subsection{Demonstrating Body Scanning Precision For Measurement}

In recent research, the $[\mathrm{TC}]^{2}$ scanner has been demonstrated to be suitable for scientific enquiry (Pandaruma, Yub, and Hunterc 2011; Gropper et al. 2012), including specially the torso region (Kim et al. 2015). However, since these studies have not demonstrated the accuracy and precision relating to the specific key measurements of this study, it is necessary to begin with a demonstration of such.

\subsubsection{Literature on Body Scanning Measurement Precision}

Stewart et al. (2010) highlight the need to adopt greater standardisation in scanning measurement protocols, focusing on variation of waist circumferences by different landmark locations. They noted that measurement protocol is important to enable comparability when variation may occur, due to changes in posture and breathing. Further to this, previous research (Y. J. Wang et al. 2011; Chi and Kennon 2006; Choi and Ashdown 2011; Mckinnon and Istook 2002) has demonstrated that the posture of the participant being scanned has a impacts significantly on the anthropometric measurements of the participants. However, these researchers focused on the statistical difference between 'scan' and 'dynamic' posture, or specifically 'between foot measurements' and therefore the degree of influence which 'natural' and 'scan' posture has on the readings is still unknown. However, these studies did not address the precision of the scan measurements themselves.

Precision within scanning has further coverage in discussion of the accuracy of the first major scan survey (Robinette and Daanen 2006). Based on the experiences of running the CAESAR survey, it is suggested without manual markers related to landmarks, scan definition of the waist has the possibility to be inaccurate compared to the accuracy developed for manual methods tied to specific landmarks and protocols. Loker et al. (2005) used body scanners to assess measurements around the abdomen and indicated variation of proportions within different size clusters. Whilst this shows the important contribution scanners offer in analysis, their reliance on the greatest protrusion of the buttocks to define hip suggests a need for further consideration of how this may contrast with manual definitions. They highlight the importance of understanding the depths between 
circumferences in assessment of the figure. This suggests recognition of the concept of measurement networks, where measurements are defined in relation to each other, as they would be within the garment (Gill 2015). Similar to the findings of Stewart et al. (2010) they indicate that changes in body size create distinct patterns of fat deposition centred on the front of the body.

\subsubsection{Body Scanner Precision Study}

While a detailed review of the various anthropometric terminology is outside the scope of this paper - See Ulijaszek and Kerr (1999) - the two most important aspects to consider are the degrees of accuracy (the degree to which a measurement differs from its 'true' value) and of precision (the degree to which a series of measurement produce similar values) (DeGarmo, Black, and Kohser 2003). Due to the way in the [TC $]^{2}$ software locates and extracts measurements of the body from relative heights with cameras calibrated to consistent and repeatable shapes (Han, Nam, and Shim 2010), the accuracy of the scanner can be considered as high, being comparable to that possible through manual measuring methods (Bougourd et al. 2000). Therefore, it is necessary to determine if the precision of the scanner is within the boundaries required for garment construction and scientific enquiry. The precision of a machine can be taken as its ability to repeatedly produce a measurement within a given range; communicated through Standard Deviation $(\sigma)$, often to $3 \sigma$ representing $99.7 \%$ Confidence in the measurement (DeGarmo, Black, and Kohser 2003). However, as Clauser et al. (1988) developed methodology and guidelines on manual measurement repeatability based on traditional manual measurements, the confidence intervals cannot be considered at the high degree of engineering (e.g. $95 \%$ or $99.7 \%$ ). This is because in manual measurements the process is dynamic and repeat measurements often occur to ensure that measurements are within defined tolerances. Therefore, for this study, the traditional precisions - outlines by Clauser et al. (1988) - shall be considered as having a Confidence Interval of $1 \sigma$; or $68 \%$ (DeGarmo, Black, and Kohser 2003).

\subsubsection{Methodology}

During the collection of scan data, a subset of those within the research (16 scans) was captured three times in the scan posture. Data was extracted from each of the three scans to Excel and used to assess repeatability of measurements using the scanner as a means to establish its potential accuracy. Analysis was undertaken using SPSS.

\subsubsection{Results}

As shown in Table 1 , the $[\mathrm{TC}]^{2}$ body scanner is of high enough precision with the selected measurement for use in anthropometric investigation, producing high enough repeatability to the 'true' measurement as required for garment construction. Additionally, the Standard Deviations generated by this study are comparable to the torso measurements of Mckinnon and Istook (2002). 
Table 1. Std. Deviation (68\% confidence Intervals) of body scanning measurement relative to clothing design requirements $(\mathrm{cm})$, based on Clauser et al. (1988)

\begin{tabular}{l|cc}
\hline Measurement & Required Repeatability & SD (16) \\
\hline [W112]_TopHipGirth & 1.10 & .58 \\
\hline [W114]_HipGirth & 1.20 & .83 \\
\hline [W143]_MaxGirthBelowWaist & 1.20 & .59 \\
\hline
\end{tabular}

\section{Methodology}

A mixed-method approach was adopted in the undertaking of this work to enable the practices of clothing measurement for the hip to be compared to hip measurements derived using body scanning. Content analysis methods (Krippendorff 2013; Neuendof 2002) were employed in the collection of hip measurement definitions from three distinct sources, classified as clothing retailers, pattern construction guides and measurement guidance/standards. Definitions were coded based on the key descriptors for measurement positioning and used to support the development of measurement extraction applied in Body Scanning analysis. A quantitative experimental Case Study was used to capture body scans of a population in both a standard Scan posture, using a [TC $]^{2}$ NX16 Scanner and a natural (LTG - Legs Together) posture; similar to that used in manual measurement methods (ISO 1989; Clauser et al. 1988; A. Stewart et al. 2011) (see Figure 1). Key measurements were extracted from the scan population and analysed with the aid of SPSS (IBM 2013) to determine the impact of hip measurement definitions and the subjects posture on the hip girths. Repeat measurements of a subset of the population were also taken to enable analysis of potential variation between repeat scans.

\subsection{Content Analysis: Definition of the Hip and Hip Girth}

The sources used in content analysis comprised retailers' websites, providing guidance on customer measurement and clothing related measurement sources within the physical and electronic library of a UK University with an established technical clothing education offering. Content analysis was extended to include key texts used to support discussion and research into measurements suitable for clothing. Measurement definitions were collected in separate Excel worksheets divided by source type; 'retailers' instructing consumers how they may establish their size relative to RTW garments; 'pattern construction' guides and 'measurement guidance/standards' (Gill and Parker 2016). Key descriptors in defining the placement of the hip measurements were identified either in relation to specific body landmarks or specific body regions and coded in each of the definitions and then used to define the groupings of definitions. Coding and grouping were undertaken by a researcher with 15 years' experience in human measurement for clothing. Because this research focused primarily on hip definitions, a waist definition comparable to that used in a number of global 
sizing surveys (Size UK and Size USA) was used as a standard so as not to complicate analysis. Analysis of the waist has been the focus of existing research, which suggests the scanner waist definition used in previous large scale sizing surveys - placing the waist girth within the region occurring above the iliac crest and below the lowest rib (see Figure 1), generally accepted as the limits defining the waist region - has been found to place the waist reliably (Gill 2011; J. Wang et al. 2003; Bigaard et al. 2005).

\subsection{Experimental Design}

Participants were selected from a convenience sample of females (aged 18+) who had agreed to be scanned to receive a detailed list of body measurements. All participants initially had their height recorded in centimetres $(+/-1 \mathrm{~mm})$ using a 'Leicester Height Measure', and weight recorded in kilograms on Marsden MPMS-250 weighing scales. Participants were scanned in their own underwear using a $[\mathrm{TC}]^{2}$ NX16 scanner.

Two scans were captured of each subject, one in each of the defined postures (Figure 1); These were classified as 'Scan' (captured in the standard $[\mathrm{TC}]^{2}$ scan posture with feet placed approximately $40 \mathrm{~cm}$ apart with hands on handholds and looking straight ahead) and LTG/ natural (as Scan, but with legs placed closer together in a posture similar to relaxed standing used in most manual methods of measurement (Clauser et al. 1988; Kemsley 1957; Beazley 1997)). Scans were repeatedly captured in both postures, until a reliable scan was obtained in each posture. Each scan was also opened and manually checked for large anomalies in landmark and measurement placement that would impact on the data. Key measurements were extracted in accordance with the points identified during the Content Analysis; see Table 3. The data was batch processed using the custom Measurement Extraction Parameters (MEP) - developed within the [TC] ${ }^{2}$ Software (TC2 2011) - to Excel and checked for consistency. Basic statistics were established and data was processed to determine waist-to-hip lengths and shape categories according to the criteria of the FFIT system (Lee et al. 2007).

\section{Results \& Analysis}

\subsection{Content Analysis: Waist and hip girth definitions}

The majority of retailers and pattern literature was found to use less specificity in defining the position of the hip with more site-specific details given in the standards; see Table 2 and Gill and Parker (2016). When the hip landmarks were less specific they often referenced the greatest protrusion of the buttocks, and in some cases indicated the hip should be taken at a set length from the waist. Whilst no guides or standards suggested set lengths, $37 \%$ of retailers indicated a length of $20 \mathrm{~cm}$ below the waist girth (see Table 2), which was also evident in analysis of the pattern literature regarding measurement guidance and methods of pattern creation (Aldrich 2008; Kunick 1984; Knowles 2005). This is suggestive of proportional expectations of the body similar to methods for representing the fashion figure (Sanderson and Gill 2014) and assertions of proportional theory from significant earlier clothing related texts (Simons 1933; Kunick 1984). The analysis of existing hip definitions 
showed four key criteria (largest, widest, buttock protrusion, and length down) that could be used to reliably define the position of the hip measurement (see Table 3). These criteria were then used to program the Measurement Extraction Parameters (MEP) within the [TC] ${ }^{2}$ body scanning software. Four hip measurements were determined and defined within the measurement extraction parameters of the $[\mathrm{TC}]^{2}$ (2011) scan analysis software; see Figure 1. This MEP was then used to extract measurements from 64 female body scans captured in two different postures using a $[\mathrm{TC}]^{2}$ NX16 scanner.

Table 2. Comparison of Hip definitions between sources; see Gill and Parker (2016)

\begin{tabular}{|c|c|c|c|c|c|c|c|c|}
\hline & \multicolumn{2}{|c|}{ Retailers } & \multicolumn{2}{|c|}{ Pattern Books } & \multicolumn{2}{|c|}{$\begin{array}{l}\text { Measurement } \\
\text { Guides and } \\
\text { Standards }\end{array}$} & \multicolumn{2}{|c|}{ Totals } \\
\hline & Count & $\%$ & Count & $\%$ & Count & $\%$ & Count & $\%$ \\
\hline 0 No Clear Guidance & 5 & 8 & 2 & 20 & 2 & 22 & 9 & 11 \\
\hline 1 Fullest/Largest Part & 22 & 37 & 3 & 30 & 1 & 11 & 26 & 33 \\
\hline 2 Set Distance from waistline & 6 & 10 & 1 & 10 & 0 & 0 & 7 & 9 \\
\hline 3 Widest part & 1 & 2 & 1 & 10 & 2 & 22 & 4 & 5 \\
\hline 4 Protrusion of the Buttocks & 11 & 18 & 0 & 0 & 0 & 0 & 11 & 14 \\
\hline 5 Relative to a Landmark & 0 & 0 & 1 & 10 & 1 & 11 & 2 & 3 \\
\hline 6 Fullest and set distance & 12 & 20 & 0 & 0 & 0 & 0 & 12 & 15 \\
\hline 7 Fullest and Buttock Protrusion & 1 & 2 & 0 & 0 & 2 & 22 & 3 & 4 \\
\hline 8 Fullest and Landmark & 0 & 0 & 1 & 10 & 1 & 11 & 2 & 3 \\
\hline 9 Set Distance from Waist and Widest & 2 & 3 & 1 & 10 & 0 & 0 & 3 & 4 \\
\hline Total: & 60 & & 10 & & 9 & & 79 & \\
\hline
\end{tabular}

Table 3. Measurement Definitions used in the $[\mathrm{TC}]^{2}$ MEP

\begin{tabular}{|c|c|c|}
\hline Name & Quick Definition & Full Definition \\
\hline Height $(\mathrm{cm})^{* *}$ & Height & Direct vertical length from the soles of the feet to the vertex of the head taken from a stadiometer \\
\hline Weight $(\mathrm{Kg})^{* *}$ & Weight & The value in $\mathrm{Kg}$ as indicated on electronic scales \\
\hline Ankle Spacing* & Ankle Spacing & The horizontal distance between the inner ankle (approx. medial malleolus) points \\
\hline Bust Girth* & Fullest Bust & Horizontal circumference taken under the arm at the level of the greatest protrusion of the bust \\
\hline Waist Girth* & $4 \mathrm{~cm}$ range from small of back & $\begin{array}{l}\text { The smallest horizontal circumference of the torso occurring within an area defined by the small } \\
\text { of the back landmark and the point } 4 \mathrm{~cm} \text { above this. }\end{array}$ \\
\hline Hip 1 Girth & Largest Full Girth & $\begin{array}{l}\text { The largest horizontal circumference of the lower torso occurring between the crotch and an area } \\
\text { of up to } 90 \% \text { of the distance to the waist }\end{array}$ \\
\hline Hip 2 Girth & Greatest Width & $\begin{array}{l}\text { The horizontal circumference of the lower torso at the largest side to side width occurring } \\
\text { between the crotch and } 90 \% \text { of the distance to the waist. }\end{array}$ \\
\hline Hip 3 Girth & Buttock Protrusion & The horizontal circumference of the lower torso, taken at the greatest protrusion of the buttocks \\
\hline Hip 4 Girth & $20 \mathrm{~cm}$ down from the waist & The horizontal circumference of the lower torso taken $20 \mathrm{~cm}$ down from the waistline. \\
\hline Waist-to-hip Lengths 1-4 & Waist-to-hip length & The difference in direct vertical height between the waist girth height and hip girth height \\
\hline
\end{tabular}




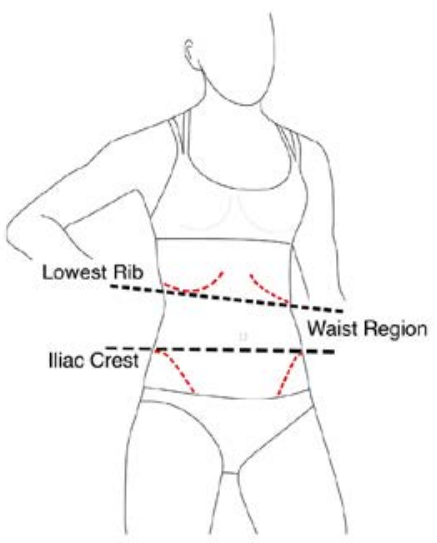

Waist Region Location

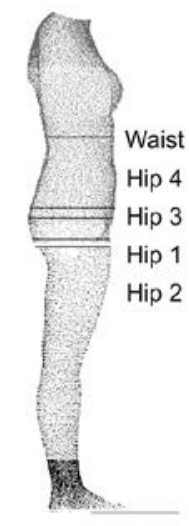

Hip Locations

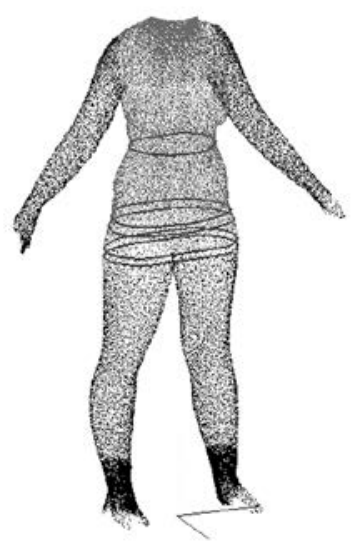

Scan Posture

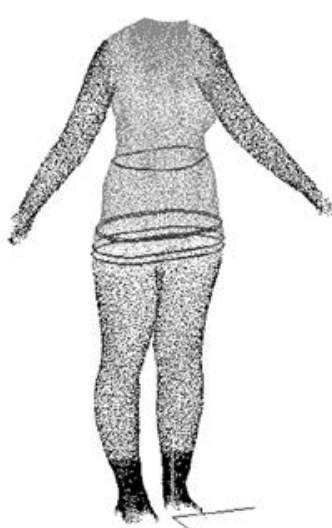

LTG (Natural) Posture

Figure 1. Scan Images showing the vertical positions of the hip girth and waist measurements on a human body and the postures used during body scanning capture ${ }^{1}$.

\subsection{Statistical Analysis of Body Scanning Measurements}

\subsubsection{Descriptive Statistics}

64 female participants were engaged within the study, aged 18-34 $(M=21.19, S D=3.44)$ with height $(\mathrm{cm})$ 143.6-183.6 $(M=166.54, S D=7.64)$ and weighing $(\mathrm{kg})$ 42-94 $(M=$ $60.48, S D=10.13)$. Taking Waist-to-Height ratios as a reliable expression of participant fitness (Ashwell, Gunn, and Gibson 2012; Schneider et al. 2010; Browning, Hsieh, and Ashwell 2010), the ratios of the sample ranged .36-.61 $(M=.44, S D=.04)$. Following the categorisation of Amen (2011), this demonstrates a wide range of bodies within the data set from clinically underweight (.35-.43) to clinically obese (.58-63). Within the sample, a variety of body shapes under the categorisation of Lee et al. (2007) were exhibited (23\% Hourglass 11\% Bottom hourglass, 48\% Rectangle, 13\% Top hourglass, 5\% Triangle), however the sample predominantly classified themselves as of white ethnicity (84\% White, 2\% Chinese, 3\% Asian, 2\% Black, 9\% Any Other Mixed background). Consequently, the sample can be considered as a representation to the wider Caucasian Female population, since ethnicity and gender has been demonstrated to influence body ratios (Singh and Luis 1995; Flegal et al. 2009). Finally, Considering the assumption of normality required for parametric statistics, all body scanner data used in this study exhibited non-significant Kolmogorov-Smirnov scores, demonstrating normality in the data set.

\subsubsection{Effect of posture on body scanning measurements}

Considering Hip Girth, a paired-samples t-test was conducted to evaluate the impact of posture (scan posture Vs. LTG) on the measured circumferences of the body at the four hip placements; see Table 3 . All measurements were statistically significant with large effect

1. Illustration based on Perez (2013), Attribution 2.0 Generic (CC BY 2.0) 
sizes $^{2}$; see Table 4. These are graphically represented within Figure 2. A mixed betweenwithin subject's analysis of variance demonstrated no statistically significant interaction between any of the Hip Girth measurements and Body Shape, height (as manually recorded with a Leicester stadiometer) to waist ratio, or Ethnicity classifications of the participants.

Table 4. Hip Girth Measurement Statistics

\begin{tabular}{llllllll}
\multicolumn{1}{c}{ Measurement } & LTD & Scan & t & $p$ & $\begin{array}{c}\bar{x} \text { Increase } \\
\text { Confidence } \\
\text { Intervals }\end{array}$ & Eta $^{2}$ \\
\hline Hip 1 Girth & $\begin{array}{l}M=98.87, \\
S D=6.67\end{array}$ & $\begin{array}{l}M=100.92, \\
S D=6.50\end{array}$ & $18.08(64)$ & $<.001$ & 2.04 & $1.81-2.27$ & 0.84 \\
\hline Hip 2 Girth & $\begin{array}{l}M=98.22, \\
S D=6.74\end{array}$ & $\begin{array}{l}M=97.51, \\
S D=6.65\end{array}$ & $13.52(64)$ & $<.001$ & 2.15 & $1.83-2.47$ & 0.74 \\
\hline Hip 3 Girth & $\begin{array}{l}M=96.95, \\
S D=6.66\end{array}$ & $\begin{array}{l}M=97.51, \\
S D=6.65\end{array}$ & $4.90(64$ & $<.001$ & 0.56 & $.33-.79$ & 0.28 \\
\hline Hip 4 Girth & $\begin{array}{l}M=97.01, \\
S D=6.34\end{array}$ & $\begin{array}{l}M=97.84, \\
S D=6.33\end{array}$ & $5.37(64)$ & $<.001$ & 0.84 & $.52-1.15$ & 0.31 \\
\hline
\end{tabular}
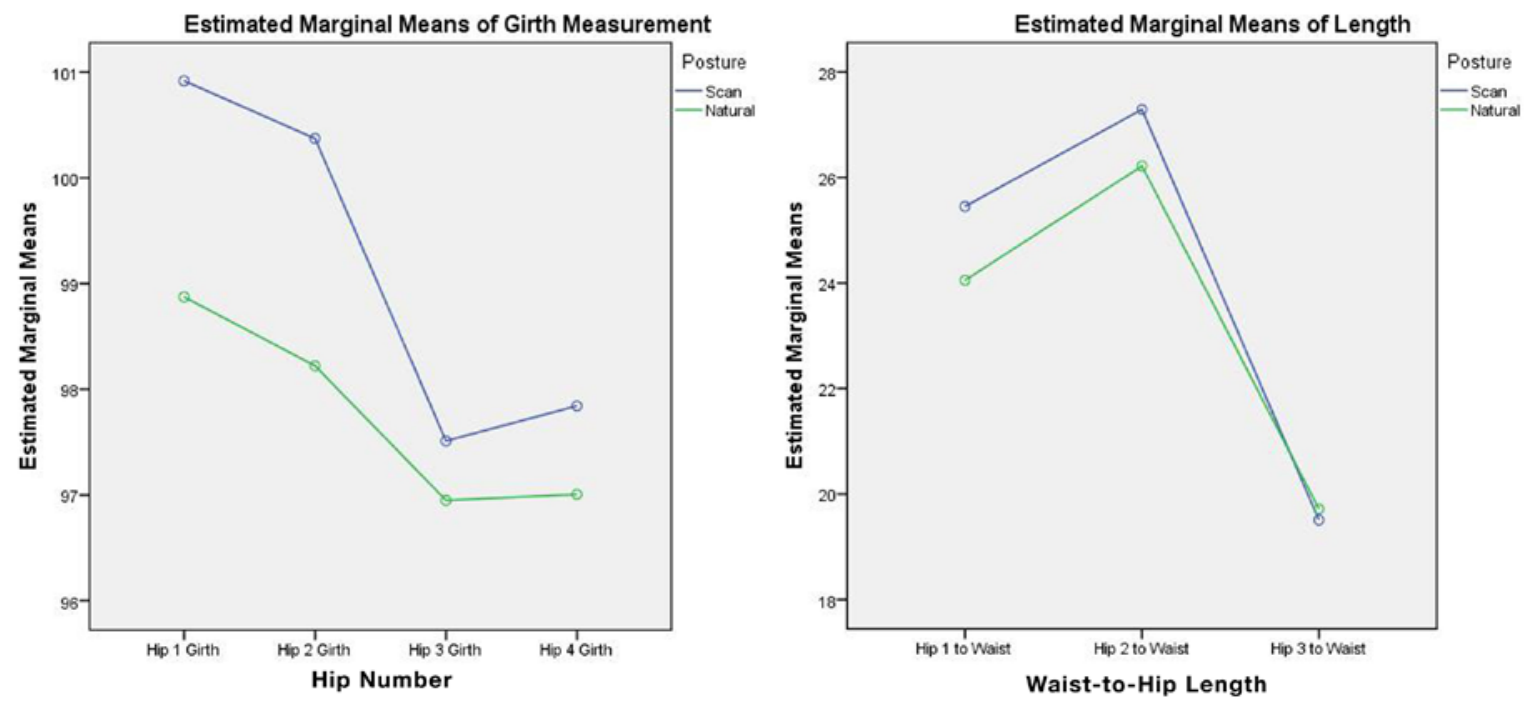

Figure 2. Line charts plotting Hip Girth for different scan postures (right) and Waist-to-hip Lengths for different scan posture (left)

Considering Waist Height, a paired-samples t-test was conducted to evaluate the impact of posture (scan posture Vs. LTG) on the height of the waist (Table 3). There was a statistically significant decrease in measurements from LTG $(M=104.26, S D=5.87)$ to Scan $(M=103.61, S D=5.71), t=-3.76(64), \rho<.001$ (two tailed). The mean decrease in measurement was -.65 with a $95 \%$ confidence interval ranging from -1.00 to -.31 . The etasquared statistic (.18) indicated a medium effect size A mixed between-within subjects

${ }^{2}$. Effect sizes indicated in relation to Cohen (1988) 
analysis of variance demonstrated that there was no statistically significant interactions between the Waist Height measurements and Body Shape, height to waist ratios or Ethnicity. Two outliers were evident, in all instances 40 has a larger hip girth than the $95 \%$ confidence intervals for the population, based on standard deviations, and in one instance so does 12 .

Considering Waist-to-hip, a paired-samples t-test was conducted to evaluate the impact of posture (scan posture Vs. LTG) on the four waist-to-hip heights (see Table 3. and Table 5). With Waist-to-hip 3 length, there was no statistically significant differences on the measurements with either scan posture or LTG posture. A mixed between-within subject's analysis of variance demonstrated that there was no statistically significant interaction between Waist-to-hip 1 Length; see Table 5.

Table 5. Waist-to-Hip Length Statistics

\begin{tabular}{|c|c|c|c|c|c|c|c|}
\hline Measurement & LTD & Scan & $\mathbf{t}$ & $p$ & $\bar{x}$ Increase & $\begin{array}{c}\text { Confidence } \\
\text { Intervals }\end{array}$ & Eta $^{2}$ \\
\hline Waist-to-hip 1 length & $\begin{array}{l}M=24.05 \\
S D=2.42\end{array}$ & $\begin{array}{l}M=25.45, \\
S D=2.60\end{array}$ & $6.10(64)$ & $<.001$ & 1.4 & $.94-1.86$ & 0.37 \\
\hline Waist-to-hip 2 length & $\begin{array}{l}M=26.22, \\
S D=2.25\end{array}$ & $\begin{array}{l}M=27.29, \\
S D=2.30\end{array}$ & $4.41(64)$ & $<.001$ & 1.07 & $.59-1.56$ & 0.24 \\
\hline
\end{tabular}

\subsubsection{Correlation of differences}

Table 6. Correlation between Change in Measurement 1 (X-Axis) and change in Measurement 2 (Y-Axis); all cases N=64; Red indicates low relationship strength, Orange medium relationship strength, and Green high relationship strength (Cohen 1988)

\begin{tabular}{|c|c|c|c|c|c|c|c|c|}
\hline & & Ankle & Hip1 Girth & Hip2 Girth & Hip3 Girth & Hip4 Girth & $\begin{array}{c}\text { Hip to } \\
\text { Waist } 1\end{array}$ & $\begin{array}{c}\text { Hip to } \\
\text { Waist } 2\end{array}$ \\
\hline \multirow{2}{*}{ Hip1 Girth } & Pearson Correlation & $.440^{*}$ & - & - & - & - & - & - \\
\hline & Sig. (2-tailed) & 0 & - & - & - & - & - & - \\
\hline \multirow{2}{*}{ Hip2 Girth } & Pearson Correlation & $.471^{* *}$ & $.833^{* *}$ & - & - & - & - & - \\
\hline & Sig. (2-tailed) & 0 & 0 & - & - & - & - & - \\
\hline \multirow{2}{*}{ Hip3 Girth } & Pearson Correlation & -0.023 & 0.122 & -0.006 & - & - & - & - \\
\hline & Sig. (2-tailed) & 0.855 & 0.338 & 0.963 & - & - & - & - \\
\hline \multirow{2}{*}{ Hip4 Girth } & Pearson Correlation & 0.104 & $.403^{* *}$ & $.313^{*}$ & -0.029 & - & - & - \\
\hline & Sig. (2-tailed) & 0.413 & 0.001 & 0.012 & 0.823 & - & - & - \\
\hline \multirow{2}{*}{ Hip to Waist 1} & Pearson Correlation & 0.179 & 0.143 & $.257^{*}$ & 0.153 & $-.572^{* *}$ & - & - \\
\hline & Sig. (2-tailed) & 0.156 & 0.258 & 0.04 & 0.228 & 0 & - & - \\
\hline \multirow{2}{*}{ Hip to Waist 2} & Pearson Correlation & 0.002 & -0.094 & $-.399^{* *}$ & $.279^{\circ}$ & $-.467^{* *}$ & $.472^{* *}$ & - \\
\hline & Sig. (2-tailed) & 0.988 & 0.462 & 0.001 & 0.026 & 0 & 0 & - \\
\hline \multirow{2}{*}{ Hip to Waist 3} & Pearson Correlation & -0.157 & -0.173 & -0.196 & $.633^{* *}$ & $-.685^{* *}$ & $.559^{* *}$ & $.563^{* *}$ \\
\hline & Sig. (2-tailed) & 0.214 & 0.173 & 0.12 & 0 & 0 & 0 & 0 \\
\hline
\end{tabular}

**. Correlation is significant at the 0.01 level (2-tailed).

*. Correlation is significant at the 0.05 level (2-tailed).

This section looks into how the change in one measurement (e.g. distance between the ankles going from LTG to Scan Posture) correlates with the other body measurements within this study. The results of Pearson product-moment correlation coefficient are presents in Table 6. Partial correlation was used to explore the relationship between the differences in measurements within the study, controlling for Waist-To-Height ratios since Figures 3 and 4 suggested non-normal body fat levels might be driving outliers. An inspection of the zero 
order correlation suggested that controlling for Waist-to-Height ratio had very little effect on the strengths of the relationships between the variables. This suggests weight will not influence how the body measurements change. Considering the influence of ankle distance variance. There was a medium, positive correlation between the Hip 1 Girth and ankle distance variance, $r=.44, n=64, \rho=<.001$, with a medium level of perceived control (19.36\% of variance) associated with the change in stance (Table 6). Additionally, there was a medium, positive correlation between the Hip 1 Girth and ankle distance variance, $r=$ $.47, n=64, \rho=<.001$, with a high level of perceived control (22.18\% of variance) associated with the change in stance.

\subsubsection{Greatest Measurement}

Considering Hip Girth, paired-sample T-tests were conducted to evaluate the difference between the four Hip girth definitions; in Scan posture. There was statistically significant differences between all pairs of measurements, with the exception of Hip Girth definitions 3 and 4; demonstrating statistical similarity. All significant results presents eta squared statistics indicating large effect sizes ( $>.14$ (Cohen 1988, 284-87) ${ }^{3}$ ) with Standard Deviations (SD) from the paired differences approaching $4-5 \mathrm{~cm}$, similar to values used to determine a whole clothing size difference (BSI 2004). Figure 3 describes the measurements for Hip Girth for both LTG and scan posture data. The greatest mean measurement of Hip Girth related to Hip 1 measurement in scan position.

${ }^{3} \mathrm{HG}^{3} 1$ Vs HG2 = .38; HG1 Vs HG3 = .84; HG1 Vs HG4 = .74; HG2 Vs HG3 = .84; HG2 Vs HG4 = .57. 


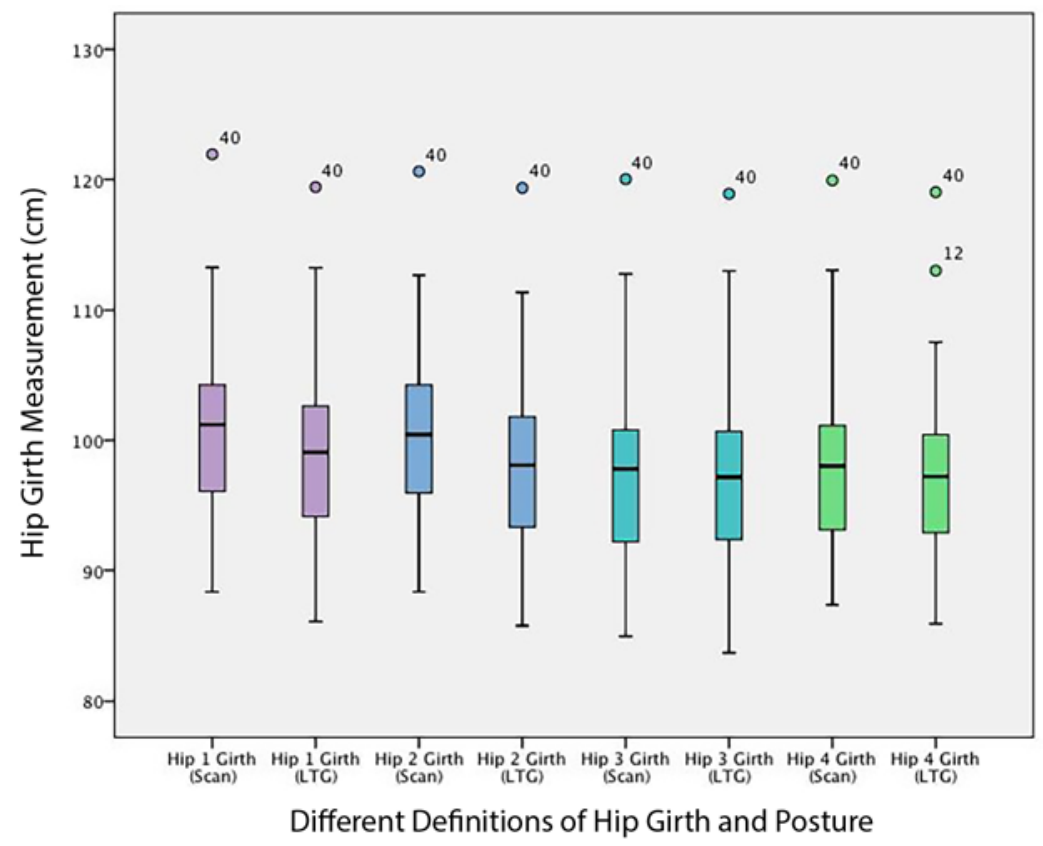

Figure 3. Box plot of Waist-to-hip Girth measurement (scan posture)

Considering waist-to-hip Lengths, paired-sample T-tests were conducted to evaluate the difference between the three Waist-to-Hip Length definitions. There were statistically significant differences between all pairs of measurements with eta squared statistics indicating large effect sizes ( $>.14$ (Cohen 1988, 284-87) ${ }^{4}$ ). Again outliers were evident; participant 35 has a considerably shorter waist to hip length than the spread of standard deviations and 13 is longer in two instances. Figure 4 describes the measurements for Waistto-hip Length, for both LTG and scan posture data. While there is a great degree of overlap within the samples, Waist-to-Hip 2 has the largest mean length, particularly in the Scan posture. The waist to hip lengths for hip girths 1 and 2 which were found to be the largest circumferences in both postures (Figure 4), suggesting a longer waist to hip length than that specified in current practice during content analysis. This also challenges the notion of a $20 \mathrm{~cm}$ waist to hip length that is often used in pattern construction methods.

${ }^{4}$ WHL1 Vs. WHL2 = .52; WHL1 Vs. WHL3 = .73; WHL2 Vs. WHL3 = .43. 


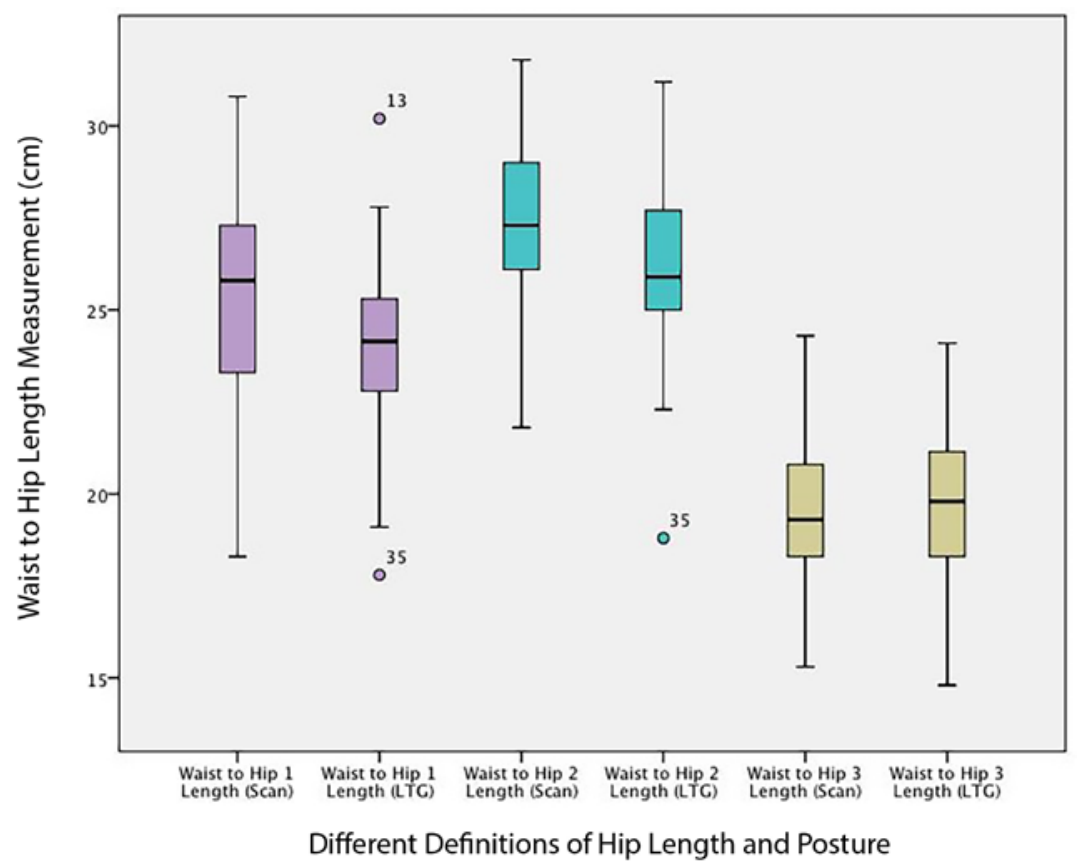

Figure 4. Box plot of Waist-to-hip Lengths (LTG posture)

\section{Discussion}

\subsection{Does posture affect circumference measurement of the hip?}

Posture was found to have a significant effect on the girth measurements of the hip and could cause an average change of $2 \mathrm{~cm}$; equal to half a clothing size. Hip 1 , the greatest circumference was found to change the most, this may be due to it being more distal than the other measurements from the hip joint and therefore subject to greater potential increase through abduction of the hip joint. Hip 3 (buttock protrusion) showed the least change between postures with an average of only $0.5 \mathrm{~cm}$ suggesting this is a more stable measurement between postures, this may explain its adoption in the ASTM (2009) standards and the ISAK documents (Stewart et al. 2011). Whilst hip 3 may offer more stability between postures, it returns a smaller measurement than Hip 1 and 2 and would therefore mislead the product developer in correctly identifying the largest circumference of the lower body. This would impact on garment fit as the extra measurement required to correctly fit over the hip would reduce the ease incorporated into the garment and would affect its ability to perform in wear and the ability to suitably control garment fit in an informed manner.

\subsection{Does posture affect the waist-to-hip length measurement?}

Waist-to-hip length reduced between the scan and LTG posture for hips 1 and 2, however, hip 3 only showed a small decrease between postures, suggesting it may be more stable or that a corresponding change to the waist position might play a part in stabilising this length. 


\subsection{Defining the most appropriate hip girth}

The largest hip definition is the most appropriate for use because garments that fit the lower body will have to pass over this girth, and the garment fit parameters including levels of ease will be determined in relation to this largest hip girth. If a smaller girth is used then this will affect the garment size and compromise the ease incorporated, making the garment too tight when worn and the ease insufficient for its requirements. Further to this hip 1 is lower on the body than the other hip measurements and suggests when a hip girth is taken at buttock protrusion or as a proportional length, the largest circumference recorded as the hip may be too high and too small. This would create stress folds at the actual hip in tighter fitting garments, potentially distorting the shape and silhouette and compromising the ease and the ability for the garment to appear and function as the designer intended.

Section 3.2.4 demonstrates that Hip Measurement 1 is the largest of the standard definitions, statistically different from Measurements $2-4$; however, the effect sizes may be considered small. Consequently, the advantage of using Hip Measurement 1 over other measurements is essential in the tailoring of close fitting garments that require precise measurements. Whilst having less visual effect on looser fitting garments using an incorrect hip circumference will affect the garment development process and the ability to control fit using existing virtual product development systems. It is essential the product developer can relate body measurements directly to the pattern and even in abstract manual methods of pattern construction girths and heights are suitably and precisely defined. Since Section 3.2.4 demonstrated no interaction between posture and measurement, with Hip 1 being consistently the largest measurement by marginal means, this outcome is applicable to persons measured in either natural [LTG] and scan postures and suggest further consideration is necessary of manual practices in defining hip girths for product development.

\subsection{Standardisation of Body Scanning Posture}

As the Medium-strength positive correlation between ankle distance and Hip 1 and 2 girths is demonstrated within Section 3.2.3, it can be implicit that the application of universal standardisation in between ankle posture is essential to allow for Body Scanning data from multiple sessions to be successfully compared. This suggests a requirement to correctly define ankle spacing in measurement data derived from body scans to ensure any effects of posture on circumference can be accounted for in analysis and application.

\subsection{Considerations of dimensions recorded from body scanning to drive clothing development}

Based on the results of this research and with consideration of the greater depth of data that can be derived from body scanning systems it is essential to consider which measurements should be recorded within data sets to be used in product development. This is important as measurement data is often extracted and used abstracted from the context of body scans and therefore considerations of posture are important. Whilst sizing often only considers a single hip, for product development both hip 1 and hip 3 should be recorded and some means of 
differentiating them indicated, i.e. hip at greatest circumference and hip at greatest buttock protrusion. Further details of ankle spacing should also accompany any hip girth data to ensure that variation in posture can be accounted for.

\subsection{Corrections to scan hip girth if using hip 3}

If practices remain where only a single definition of the hip is used and this is placed in relation to maximum posterior buttock protrusion, it may be necessary to correct any recorded depths to reflect the actual height of the hip. It is suggested from this research that the buttock protrusion can be used to define the hip but with most participants the lengths from waist to hip should be increased by between $2-3 \mathrm{~cm}$ to reflect the actual height of the larger hip circumference This becomes more important with closer fitting garments and those which taper or have a tailored fit following the contours of the body.

\subsection{Limitations}

Whilst the population sample within this study can be considered generalizable to the wider population (see Section 3.2.1), the younger demographic of this research relative to the UK population would be expected to have a defined waist in contrast to the less defined waist of an older demographic (J. Wang et al. 2003). This is recognised as a limitation in representing these findings to the population as a whole. However, this well-defined waist should reduce variation in waist placement using the body scanner, as the geometry of the body usually provides a clear demarcation of the waist shaping. Additionally, the use of only female participants limits the insights gained on the effects of posture on body measurements, especially since gender, age and other similar variables play a significant role (Rosenbaum et al. 1996).

\section{Conclusions}

This research indicates that 1) body scanning posture affects where in the hip region (crotch to waist) the hip circumference will be placed and the size of the hip measurement defined, 2) this can have a significant impact on hip girth measurements, and 3) posture may affect other key dimensions required for clothing product development and sizing categorisation; e.g. waist and bust. Our results indicate that it is imperative that studies using human measurement are always explicit in their measurement definition, as this variable can influence the resulting measurement amount. There is varied definition and details related to the hip and its position in sizing and product development practice, however, body scanning can be seen as an effective tool for defining multiple locations of the hip. Singular hip definitions (especially related to posterior buttock protrusion) may not return values that are optimal to drive product development, especially when fit will need to be precisely controlled. This also raises issues of the comparability of measurements between different scan data sets using the same names but different hip definitions. Further to this, greater research needs to be conducted on the measurement and communication of body scanning accuracy within the fields of anthropometric research. 
The strengths of body scanning and the results of this study indicate it may be necessary to determine at least two measurements of the hip, one in accordance with the largest lower body dimension and the other in line with buttock protrusion. This would enable clothing to better reflect the shape of the lower torso and fit better to varied body shapes and proportions. Hip definitions should always be explicitly stated and recognition given to how deviations from accepted measurement definitions are catalogued.

\subsection{Further Research}

To create more consistency in consumer collected data without the aid of body scanning technology it may be more appropriate to determine the self-measured hip in relation to the widest point on the lower body, rather than over the greatest posterior protrusion of the buttocks. If clear guidance is given for self-measurement it may be suitable to ask the consumer to measure at both points and input the largest value, though this still does not overcome potential variation in segment lengths, between circumferences, which is difficult to accurately take without automated measurement tools. Fundamentally methods of defining the hip measurement need to have greater sensitivity to the application of that data within methods of product development; this may require greater standardisation between sources and clear recognition that existing methods may not be capturing the largest girth.

This area needs to be further developed to establish the effects of posture on a larger population and consider body shape as well as potential differences caused through ageing and changed fat deposition. Without standardisation of scan postures used to create measurement sets for product development and a clear definition of the hip or hip region it will be difficult to ensure measurements defined as hip will truly reflect the right dimensions to drive product development.

\section{Acknowledgements}

Kathryn Brownbridge, Paula Wren, Jon Spragg, Steve Hayes, Peter Royle, and the technical support staff at the Hollings faculty of Manchester Metropolitan University who helped with data collection and tools for analysis.

\section{References}

Aldrich, W. 2008. Metric Pattern Cutting for Women's Wear. 5th ed. Oxford, UK: Blackwell Publishing.

Alexander, M., G.R. Pisut, and A Ivanescu. 2012. 'Investigating Women’s plus-Size Body Measurements and Hip Shape Variation Based on SizeUSA Data'. International Journal of Fashion Design, Technology and Education 5 (1): 3-12.

Amen, Daniel G. 2011. The Amen Solution: The Brain Healthy Way to Lose Weight and Keep It Off: Daniel G. Amen: Amazon.com: Books. Reprinted. USA: Harmony.

Ashdown, S.P., and H. Na. 2008. 'Comparison of 3-D Body Scan Data to Quantify UpperBody Postural Variation in Older and Younger Women'. Clothing and Textiles Research Journal 26 (4): 292-307. 
Ashwell, M., P. Gunn, and S. Gibson. 2012. 'Waist-to-Height Ratio Is a Better Screening Tool than Waist Circumference and BMI for Adult Cardiometabolic Risk Factors: Systematic Review and Meta-Analysis’. Obesity Reviews 13: 275-86.

ASTM. 2009. D5219-09: Standard Terminology Relating to Body Dimensions for Apparel Sizing. Standard.

Beazley, A. 1997. 'Size and Fit: Procedures in Undertaking a Survey of Body Measurements - Part 1'. Journal of Fashion Marketing and Management 2 (1): 55-85.

Beazley, A., and T. Bond. 2003. Computer-Aided Pattern Design and Product Development. Oxford, UK: Blackwell Publishing.

Bigaard, J., I. Spanggaard, B. Lykke Thomsen, K. Overvad, and A. Tjonneland. 2005. 'SelfReported and Technician-Measured Waist Circumferences Differ in Middle-Aged Men and Women'. The Journal of Nutrition 135 (9): 2263-70.

Bonnechère, B, B Jansen, P Salvia, H Bouzahouene, V Sholukha, J Cornelis, M Rooze, and S Van Sint Jan. 2014. 'Determination of the Precision and Accuracy of Morphological Measurements Using the Kinect $^{\mathrm{TM}}$ Sensor: Comparison with Standard Stereophotogrammetry'. Ergonomics 57 (4). Taylor \& Francis: 622-31. doi:10.1080/00140139.2014.884246.

Bougourd, J. P., L. Dekker, P. Grant Ross, and J. P. Ward. 2000. ‘A Comparison of Women’s Sizing by 3D Electronic Scanning and Traditional Anthropometry'. Journal of The Textile Institute 91 (2): 163-73. doi:10.1080/00405000008659536.

Browning, Lucy M, Shiun Dong Hsieh, and Margaret Ashwell. 2010. 'A Systematic Review of Waist-to-Height Ratio as a Screening Tool for the Prediction of Cardiovascular Disease and Diabetes: 0.5 Could Be a Suitable Global Boundary Value'. Nutrition Research Reviews 23 (2). Cambridge University Press: 247-69. doi:10.1017/S0954422410000144.

BSI. 2002. BS EN 13402-2:2002, Size Designation of Clothes - Part 2: Primary and Secondary Dimensions. Standard. London, UK: British Standards Institute.

—. 2004. 'BS EN 13402-3:2004, Size Designation of Clothes - Part 3: Measurements and Intervals'. British Standard. London, UK: British Standards Institute.

- 2010. BS EN ISO 20685:2010: 3-D Scanning Methodologies for Internationally Compatible Anthropometric Databases. Standard. London, UK: British Standards Institute.

Bye, Elizabeth., Karen. L. LaBat, and Marilyn. R. Delong. 2006. 'Analysis of Body Measurement Systems for Apparel'. Clothing and Textiles Research Journal 24 (2): 6679. doi:10.1177/0887302X0602400202.

Chi, Liu, and Richard Kennon. 2006. 'Body Scanning of Dynamic Posture'. International Journal of Clothing Science and Technology 18 (3): 166-78. doi:10.1108/09556220610657934.

Choi, S., and S.P. Ashdown. 2011. '3D Body Scan Analysis of Dimensional Change in Lower Body Measurements for Active Body Positions’. Textile Research Journal 81 (1): 81-93. doi:10.1177/0040517510377822.

Clauser, C.E., I.O. Tebbetts, B. Bradtmiller, J.T. McConville, and C.C. Gordon. 1988. Measurers Handbook: US Army Anthropometric Survey. Natick, MA: US Army Natick Research Development \& Engineering Centre (TR-88/043).

Cohen, Jacob J.W. 1988. Statistical Power Analysis for the Behavioral Sciences. 2nd ed. 
Hillsdale, USA: Lawrence Erlbaum Associates.

Daniell, Nathan, Tim Olds, and Grant Tomkinson. 2010. 'The Importance of Site Location for Girth Measurements'. Journal of Sports Sciences 28 (7): 751-57. doi:10.1080/02640411003645703.

DeGarmo, E Paul, J T Black, and Ronald A Kohser. 2003. Materials and Processes in Manufacturing. BOOK. 9th ed. Vol. Ninth. USA: John Wiley \& Sons.

Flegal, Katherine M, John A Shepherd, Anne C Looker, Barry I Graubard, Lori G Borrud, Cynthia L Ogden, Tamara B Harris, James E Everhart, and Nathaniel Schenker. 2009. 'Comparisons of Percentage Body Fat, Body Mass Index, Waist Circumference, and Waist-Stature Ratio in Adults'. The American Journal of Clinical Nutrition 89 (2): 500508. doi:10.3945/ajcn.2008.26847.

Gill, Simeon. 2009. 'Determination of Functional Ease Allowances Using Anthropometric Measurement for Application in Pattern Construction'. Manchester Metropolitan University.

—. 2011. 'Scanner Waist'. Bodyscanning. Accessed March 282014. http://bodyscanning.wordpress.com/scanner-waist/.

2015. 'A Review of Research and Innovation in Garment Sizing, Prototyping and Fitting'. Textile Progress 47 (1): 1-85. doi:10.1080/00405167.2015.1023512.

Gill, Simeon, and Kathryn Brownbridge. 2013. 'The Myth of Standard Size'. In Beauty 3rd Global Conference. Oxford, UK: Harris Manchester College.

Gill, Simeon, and N. Chadwick. 2009. 'Determination of Ease Allowances Included in Pattern Construction Methods'. International Journal of Fashion Design, Technology and Education 2 (1): 23-21. doi:10.1080/17543260903018990.

Gill, Simeon, and Christopher J. Parker. 2016. Variation in Defining the Hip Circumference for Clothing Applications. ADE1601. Manchester, UK. doi:10.13140/RG.2.1.3450.0087.

Gill, Simeon, Christopher J. Parker, Steve Hayes, Paula Wren, and Anastasiia Panchenko. 2014. 'The True Height of the Waist: Explorations of Automated Body Scanner Waist Definitions of the TC2 Scanner'. In 5th International Conference and Exhibition on 3D Body Scanning Technologies, 55-65. Lugano, Switzerland: Hometrica Consulting. doi:10.15221/14.055.

Gropper, Sareen S, Karla P Simmons, Lenda Jo Connell, and Pamela V Ulrich. 2012. 'Changes in Body Weight, Composition, and Shape: A 4-Year Study of College Students'. Applied Physiology, Nutrition, and Metabolism = Physiologie Appliquée, Nutrition et Métabolisme 37 (6). NRC Research Press: 1118-23. doi:10.1139/h2012139.

Haggar, A. 2004. Pattern Cutting for Lingerie, Beachwear and Leisurewear. Oxford, UK: Blackwell Publishing.

Han, H., Y. Nam, and S.H. Shim. 2010. 'Algorithms of the Automatic Landmark Identification for Various Torso Shapes'. International Journal of Clothing Science and Technology 22 (5): 343-57.

Heuberger, R., T. Domina, and M. MacGillivray. 2008. 'Body Scanning as a New Anthropometric Measurement Tool for Health-Risk Assessment'. International Journal of Consumer Studies 32 (1): 34-40.

IBM. 2013. 'SPSS'. USA: IBM Ltd. 
ISO. 1989. Garment Construction and Anthropometric Surveys - Body Dimensions. Standard. - 2010. 7250-1:2010: Basic Human Body Measurements for Technological Design Part 1: Body Measurement Definitions and Landmarks. Standard. International Standards Organisation.

Kapandji, I.A. 1987. The Physiology of the Joints, Volume 3 The Trunk and the Vertebral Column. Edinburgh: Churchill Livingstone.

Kemsley, W.F.F. 1957. Women's Measurements and Sizes. London, UK: Joint Clothing Council Ltd, H.M.S.O.

Kim, Dong-Eun, Karen LaBat, Elizabeth Bye, MyungHee Sohn, and Karen Ryan. 2015. 'A Study of Scan Garment Accuracy and Reliability'. The Journal of The Textile Institute 106 (8): 853-61. doi:10.1080/00405000.2014.949502.

Knowles, L.A. 2005. The Practical Guide to Patternmaking for Fashion Designers, Juniors, Misses and Women. New York, USA: Fairchild Publications.

Kouchi, M., and M. Mochimaru. 2011. 'Errors in Landmarking and the Evaluation of the Accuracy of Traditional and 3d Anthropometry’. Applied Ergonomics 42 (3): 518-527. doi:10.1016/j.apergo.2010.09.011.

Krippendorff, K. 2013. Content Analysis. 3rd ed. CA, USA: Sage Publications.

Kunick, P. 1984. Modern Sizing and Pattern Making for Womens, Mens and Childrens Garments. London, UK: Philip Kunick.

Lee, J.Y., C.L. Istook, Y.J. Nam, and S.M. Park. 2007. 'Comparison of Body Shape between USA and Korean Women'. International Journal of Clothing Science and Technology 19 (5): 374-91.

Li, Zhizhong, Chien-Chi Chang, Patrick G Dempsey, Lusha Ouyang, and Jiyang Duan. 2008. 'Validation of a Three-Dimensional Hand Scanning and Dimension Extraction Method with Dimension Data'. Ergonomics 51 (11). Taylor \& Francis: 1672-92. doi:10.1080/00140130802287280.

Loker, Suzanne, Susan Ashdown, and Katherine Schoenfelder. 2005. 'Size-Specific Analysis of Body Scan Data to Improve Apparel Fit'. Journal of Textile and Apparel Technology and Management 4 (3): 16-33.

MacDonald, M.N. 2010. Principles of Flat-Pattern Design. 4th ed. New York, USA: Fairchild Publications.

McKinney, E.C., E. Bye, and K. Labat. 2012. 'Building Patternmaking Theory: A Case Study of Published Patternmaking Practice for Pants'. International Journal of Fashion Design, Technology and Education 5 (3): 153-67.

Mckinnon, Lashawnda, and Cynthia L. Istook. 2002. 'Body Scanning: The Effects of Subject Respiration and Foot Positioning on the Data Integrity of Scanned Measurements'. Journal of Fashion Marketing and Management 6 (2): 103-21.

Neuendof, K.A. 2002. The Content Analysis Guidebook. CA, USA: Sage Publications.

Pandaruma, Reena, Winnie Yub, and Lawrance Hunterc. 2011. '3-D Breast Anthropometry of plus-Sized Women in South Africa'. Ergonomics 54 (9): 866-75. doi:10.1080/00140139.2011.597515.

Paquette, Steven., David. Brantley, Brian. D. Corner, Peng. Li, and Thomas. Oliver. 2000. 'Automated Extraction of Anthropometric Data from 3D Images'. In Proceedings of the IEA 2000/HFES 2000 Congress, 727-30. 
Perez, S. 2013. 'All Women Lifeguard Tournament 2013’. Photo. Flickr. Online: Flickr. Accessed June 18 2014. https://flic.kr/p/fmVCX3.

Pheasant, S. 1990. Anthropometrics: An Introduction. 2nd ed. Milton Keynes, UK: British Standards Institute.

Robinette, K.M., and H.A.M. Daanen. 2006. 'Precision of the CAESAR Sacn-Extracted Measurements'. Applied Ergonomics 37 (3): 259-65.

Roebuck, J. 1995. Anthropometric Methods: Designing to Fit the Human Body. Monica, CA: Human Factors and Ergonomics Society.

Rosenbaum, M, M Nicolson, J Hirsch, S B Heymsfield, D Gallagher, F Chu, and R L Leibel. 1996. 'Effects of Gender, Body Composition, and Menopause on Plasma Concentrations of Leptin.' The Journal of Clinical Endocrinology and Metabolism 81 (9). American Public Health Association: 3424-27. doi:10.1210/jcem.81.9.8784109.

Sanderson, R, and Simeon Gill. 2014. 'Turning Figure Drawing on Its Head'. In The Power of Fashion: 16th Annual IFFTI Conference. Bunka Gakuen, Japan: Bunka Gakuen University.

Schneider, Harald J, Nele Friedrich, Jens Klotsche, Lars Pieper, Matthias Nauck, Ulrich John, Marcus Dörr, et al. 2010. 'The Predictive Value of Different Measures of Obesity for Incident Cardiovascular Events and Mortality.' The Journal of Clinical Endocrinology and Metabolism 95 (4). Endocrine Society: 1777-85.

Simmons, Karla Peavy. 2001. 'Body Measurement Techniques: A Comparison of ThreeDimensional Body Scanning and Physical Anthropometric Methods’. North Carolina State University.

Simons, Harry. 1933. The Science of Human Proportions. New York: Clothing Designer CO., Inc.

Singh, D, and S Luis. 1995. 'Ethnic and Gender Consensus for the Effect of Waist-to-Hip Ratio on Judgment of Women's Attractiveness'. Human Nature 6 (1): 51-65. doi:10.1007/BF02734135.

Stewart, A. D., A. M. Nevill, R. Stephen, and J. Young. 2010. 'Waist Size and Shape Assessed by 3D Photonic Scanning'. International Journal of Body Composition Research 8 (4): 123-30.

Stewart, A., M. Marfell-Jones, T. Olds, and H. de Ridder. 2011. International Standards for Anthropometric Assessment. Underdale, AUS: The International Society for the Advancement of Kinanthropometry.

TC2. 2011. '[TC] ', Cary, NC, USA: TC2.

Tyler, D, A Mitchell, and Simeon Gill. 2012. 'Recent Advances in Garment Manufacturing Technology; Joining Techniques, 3D Body Scanning and Garment Design'. In The Global Textile and Clothing Industry, edited by R Shishoo, 131-70. Cambridge, UK: Woodhead Publishing.

Ulijaszek, Stanley J., and Deborah A. Kerr. 1999. 'Anthropometric Measurement Error and the Assessment of Nutritional Status'. British Journal of Nutrition 82 (3). Cambridge University Press: 165-77. doi:10.1017/S0007114599001348.

Wang, J., J.C. Thornton, S. Bari, B. Wiulliamson, D. Gallagher, S.B. Heymsfield, M. Horlick, et al. 2003. 'Comparisons of Waist Circumferences Measured at 4 Sites'. American Journal of Clinical Nutrition 77 (2): 379-84. 
Wang, Y. J., P. Y. Mok, Y. Li, and Y. L. Kwok. 2011. 'Body Measurements of Chinese Males in Dynamic Postures and Application’. Applied Ergonomics 42 (6): 900-912. doi:10.1016/j.apergo.2011.02.006. 